\title{
Schwannoma quístico del porta hepatis
}

\author{
Oliver D. Maida M. ${ }^{1,2}$, Xavier A. De Aretxabala U. ${ }^{1,2}$, \\ Giancarlo Schiappacasse F., ${ }^{2,3}$ y Leonor A. Castiblanco G. ${ }^{2,4}$
}

\footnotetext{
'Servicio de Cirugía Clínica Alemana.

¿Universidad del Desarrollo ${ }^{3}$ Servicio de Radiología Clínica Alemana. ${ }^{4}$ Departamento de Anatomía Patológica Clínica Alemana.

Recibido el 8 de abril de 2019 y aceptado para publicación e

2 de octubre de 2019 .
}

Correspondencia a:

Dr. Oliver D. Maida M. oliver_maida@hotmail.com

\section{Cystic Schwannoma of Porta Hepatis}

Introduction: Bile duct schwannoma is an extremely rare condition. We report a 45 years old male patient in whom during a rutinary ultrasound exam a liver cyst was detected. Magnetic resonance confirmed lesion and the histopathology of the resected specimen was a benign schwannoma proven by positive inmunoreaction to vimentina.

Key words: biliar duct; Schwannoma; benign tumor; liver tumor; liver.

\section{Resumen}

Introducción: Los Schwannomas de la vía biliar corresponden a una entidad extremadamente infrecuente, habiéndose reportado solo escasos casos en la literatura. Caso Clínico: El presente paciente corresponde a un hombre de 45 años, a quien durante examen rutinario se le pesquisa una lesión quística hepática. La resonancia magnética confirmó la lesión, y la biopsia de la pieza resecada diagnosticó la existencia de un Schwannoma benigno con marcadores positivos para vimentina y proteína S-100.

Palabras clave: vía biliar; Schwannoma; tumor benigno; tumor hepático; hígado.

\section{Introducción}

Los Schwannomas son tumores gliales derivados de las células de Schwann, se caracterizan por ser benignos, encapsulados y de crecimiento lento. Suelen ocurrir en la cabeza, cuello, retroperitoneo, mediastino y pelvis, y en asociación con enfermedades sistémicas como la neurofibromatosis tipo $2^{1}$. Sin embargo, los Schwannomas que se originan del parénquima hepático son extremadamente infrecuentes.

Dada su forma de presentación y baja frecuencia, resulta difícil en muchos casos realizar el diagnóstico sólo con imágenes, aunque en algunos casos su morfología es característica. Para los restantes casos se requiere de la resección completa o bien biopsia para establecer el diagnóstico definitivo.

Presentamos a continuación el caso de un Schwannoma y la revisión de la literatura.

\section{Reporte del caso}

Paciente masculino de 45 años sin antecedentes de importancia, quien durante un examen de salud preventivo se pesquisó mediante una ecografía abdominal un quiste complejo a nivel hepático (Figura 1). Con la finalidad de caracterizarla de mejor manera, se solicita resonancia magnética (RM) de abdomen, que muestra lesión quística bien delimitada localizada en relación al hilio hepático y/o lóbulo derecho, de paredes finas con componente exofítico en su interior que mide aproximadamente $47 \mathrm{~mm}$ de diámetro (Figuras 2 y 3 ).

Frente a la imagen obtenida, se plantean los siguientes diagnósticos diferenciales: quiste hidatídico hepático, quiste hepático complejo, cistoadenoma biliar, neoplasia mucinosa quística o neoplasia papilar intraductal del conducto biliar. La serología IgG para hidatidosis que resultó negativa y ante la dificultad de progresar en su diagnóstico se decide operar al paciente para realizar la resección y biopsia de la lesión.

Mediante una laparotomía transversa se explora la cavidad abdominal, pesquisando la existencia de una lesión quística ubicada en el pedículo hepático en íntimo contacto con la vena porta en su pared posterior y con la vía biliar en su pared anterior. Mediante disección se logra aislar completamente el quiste de las estructuras del pedículo y del pa- 

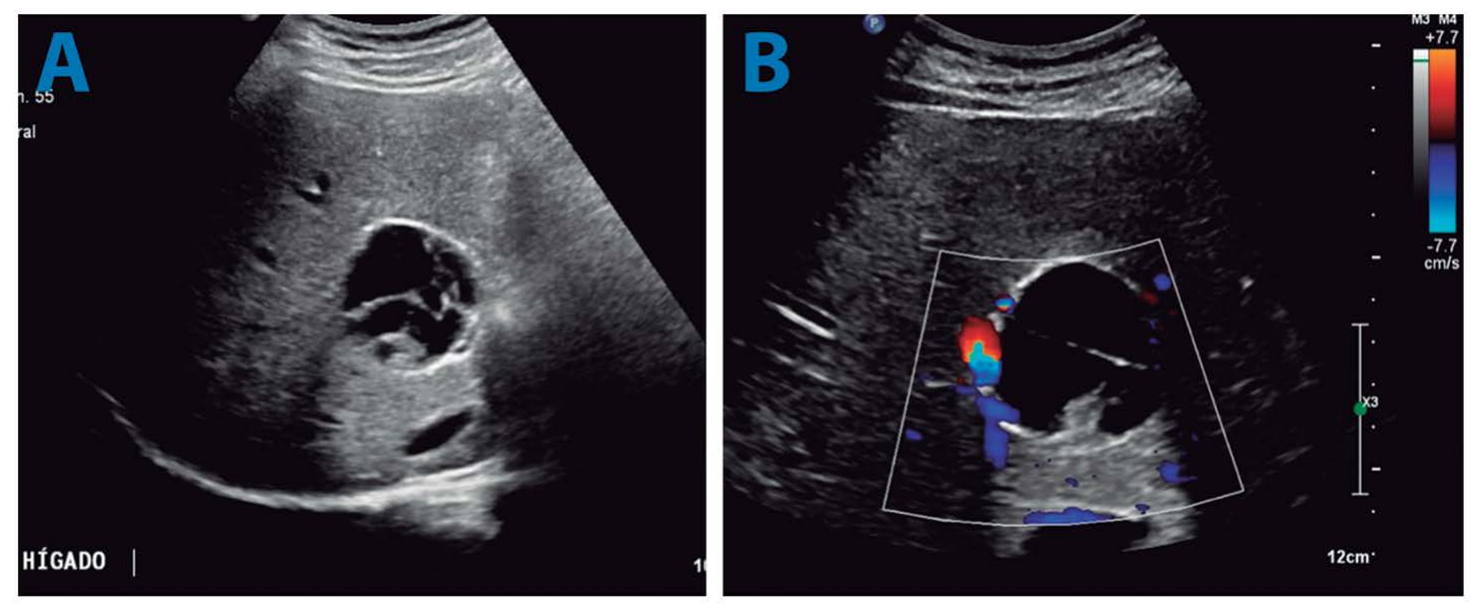

rénquima hepático. Con la finalidad de completar la resección y descartar la presencia de compromiso de la vía biliar se realiza colecistectomía y colangiografía transcística (Figura 4), que muestra indemnidad de la vía biliar y ausencia de comunicación entre el quiste y ésta.

Paciente evoluciona de manera favorable y se da de alta al $6^{\circ}$ día postoperatorio.

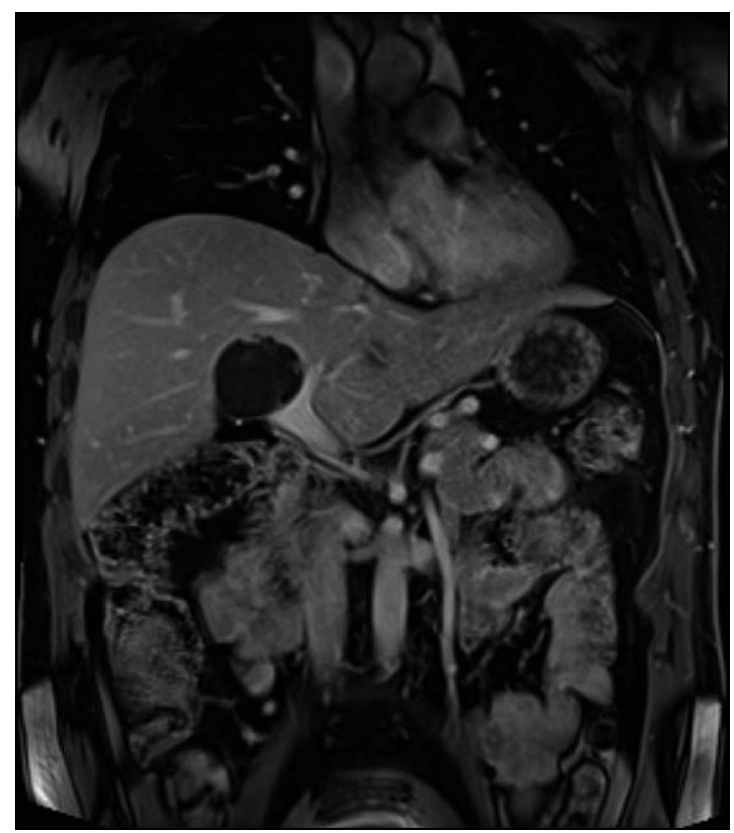

Figura 3. Resonancia magnética abdominal (secuencia coronal T1 fat-sat con contraste intravenoso): Se observa lesión quística con fina cápsula periférica y algunos finos septos en su interior que muestran escaso realce con el contraste intravenoso. Nótese que la lesión se encuentra en relación a la porta principal y que comprime parcialmente la rama portal derecha.
En la biopsia definitiva se informan células fusiformes con núcleos elongados de bordes romos compatibles con Schwannoma con degeneración quística (Figuras 5 y 6).

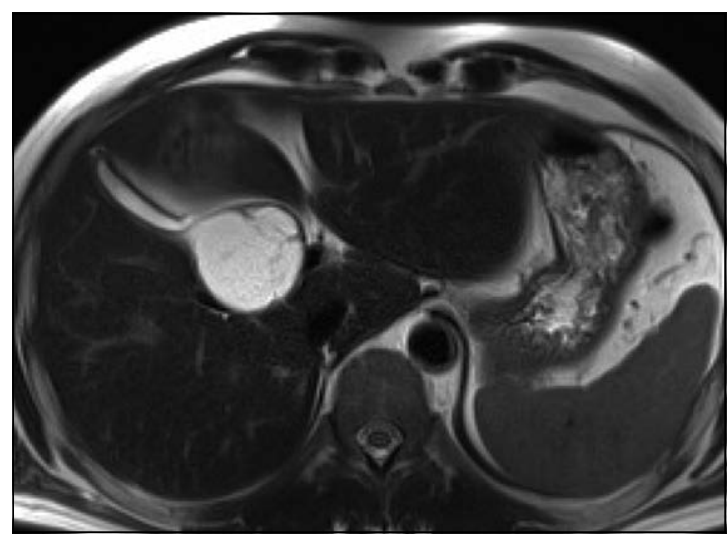

Figura 2. Resonancia magnética abdominal (secuencia axial T2 HASTE): Se observa lesión quística encapsulada localizada en relación al hilio hepático y en contacto con el lóbulo hepático derecho, que muestra algunos septos finos en su interior.

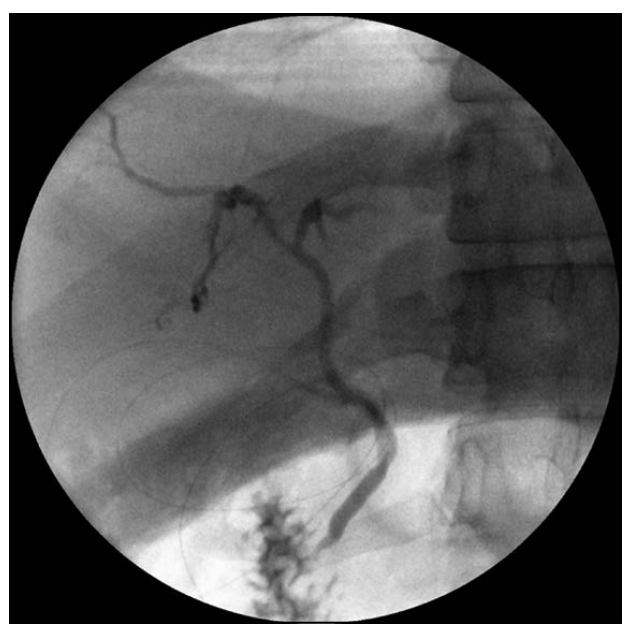

Figura 4. Colangiografía intraoperatoria: Se observa leve desplazamiento de la vía biliar extrahepática (conducto hepático común) secundario a efecto de masa por la lesión quística descrita en ecografía y resonancia magnética, sin comunicación a ésta. 


\section{CASOS CLínICOS}

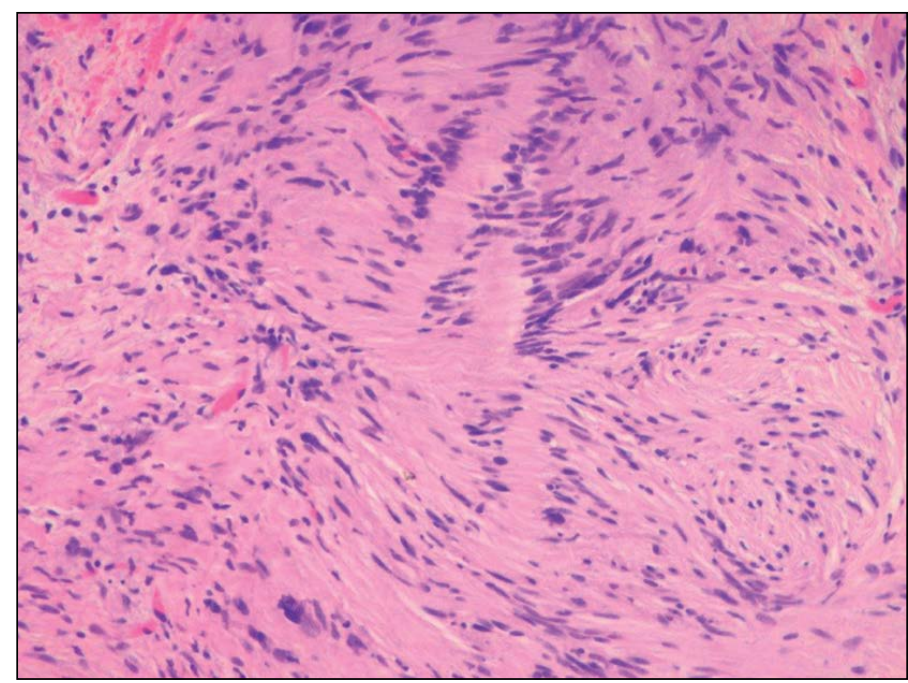

Figura 5. Células fusiformes con núcleos elongados que forman empalizadas con prolongaciones citoplasmáticas hacia el centro formando cuerpos de Verocay (zonas Antoni A).

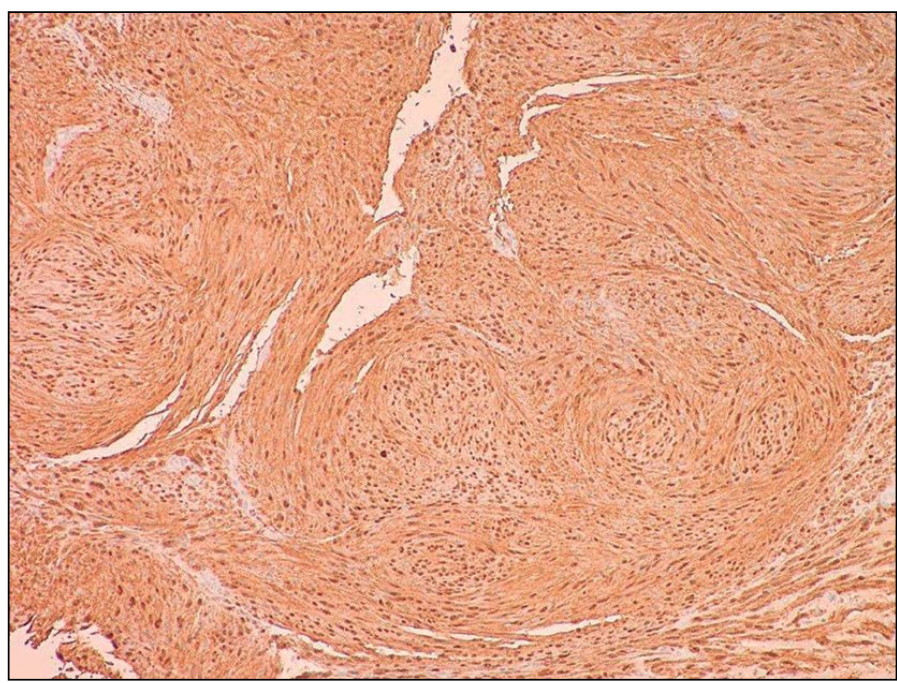

Figura 6. Tinción inmunohistoquímica para S100 positiva difusa en núcleos y citoplasma.
Tabla 1. Casos de Schwannomas biliares reportados en la literatura (se reporta un caso por autor)

\begin{tabular}{|c|c|c|c|}
\hline Referencia & Edad & Sexo & Localización del tumor \\
\hline Whisnant $1974^{3}$ & 15 & $\mathrm{~F}$ & Colédoco distal \\
\hline Balart $1983^{4}$ & 56 & $\mathrm{~F}$ & Conducto hepático común \\
\hline Oden $1995^{5}$ & 40 & $\mathrm{~F}$ & Colédoco \\
\hline Jakobs $2003^{6}$ & 37 & M & Conducto hepático común \\
\hline Honjo $2003^{7}$ & 48 & $\mathrm{~F}$ & Colédoco \\
\hline Otani $2005^{8}$ & 59 & $\mathrm{~F}$ & Conducto biliar intrahepático \\
\hline Park $2006^{9}$ & 53 & $\mathrm{~F}$ & Porta hepatis \\
\hline Vyas $2006^{10}$ & 29 & $\mathrm{~F}$ & Colédoco \\
\hline Kamani $2007^{11}$ & 39 & $\mathrm{~F}$ & Conducto hepático común proximal \\
\hline Fenoglio $2007^{12}$ & 41 & $\mathrm{~F}$ & Colédoco medio \\
\hline Jung $2007^{13}$ & 64 & $\mathrm{~F}$ & Colédoco proximal \\
\hline Madhusudhan $2009^{14}$ & 46 & M & Conducto biliar intrahepático \\
\hline Kulkarni $2009^{15}$ & 38 & M & Porta hepatis \\
\hline De Sena $2009^{16}$ & 58 & $\mathrm{~F}$ & Vía biliar extrahepática \\
\hline Parameshwarappa $2010^{17}$ & 38 & M & Colédoco \\
\hline Panait $2011^{18}$ & 54 & $\mathrm{~F}$ & Porta hepatis \\
\hline Fonseca $2012^{19}$ & 24 & $\mathrm{M}$ & Conducto hepático común proximal \\
\hline Campos $2015^{20}$ & 62 & M & Conducto hepático común \\
\hline
\end{tabular}

\section{Discusión}

Los Schwannomas son tumores benignos generados de las células de Schwann, el componente principal de las vainas neurales. Son raros dentro del sistema digestivo y, generalmente, se encuentran en el estómago, colon y esófago. Los Schwannomas biliares son extremadamente infrecuentes a pesar de que dicha región anatómica tiene abundante inervación simpática y parasimpática. El origen de los Schwannomas de ubicación hepática o biliar serían los plexos simpáticos y parasimpáticos del pedículo hepáticos, los cuales posteriormente se distribuyen a lo largo de las arterias y vasos portales en el tejido conectivo interlobar ${ }^{2}$.

Revisando la base de datos de Medline encontramos solo 18 reportes de estos tumores publicados (Tabla 1). Se reporta un ligero predominio femenino (4:3), una media de edad de 44 años (rango 15-64) al momento del diagnóstico y una distribución homogénea dentro del árbol biliar (Figura 7).

Clínicamente, los Schwannomas pueden manifestarse por dolor abdominal vago, ictericia o simplemente como una lesión hepática asintomática en los estudios de imágenes. Actualmente no hay evidencia que sugiera el potencial maligno de estos tumores ${ }^{19}$.

El diagnóstico diferencial tiene que realizarse con otras masas solitarias de aspecto multiquístico como: quiste hidatídico, neoplasia mucinosa quística $(\mathrm{MCN})$ o neoplasia papilar intraductal del conducto biliar (IPNB). 
El quiste hidatídico, enfermedad prevalente en Chile, es una enfermedad parasitaria originada por el parásito Echinococcus granulosus. A pesar de que esta enfermedad puede ocurrir en cualquier parte del cuerpo, se manifiesta de preferencia en el hígado. Suelen acompañarse de calcificaciones a diferencia del Schwannoma.

El examen imagenológico en el caso del quiste hidatídico hepático suele ser la ecografía que se puede complementar con tomografía computada o resonancia magnética y el diagnóstico serológico puede ser por enzimo-inmunoanálisis (ELISA) como examen de cribado y western blot como examen de confirmación, buscando anticuerpos IgG, IgM e IgA. En el caso de la IgE nos permite hacer el seguimiento, ya que 30 días después de la resección quirúrgica la misma debería estar negativa.

En el caso de IPNB, a pesar de que estas suelen ser masas quísticas en forma de racimo de uva, se diferencian de los Schwannomas por tener comunicación con la vía biliar, dilatación de la vía biliar y proyecciones papilares de la misma.

La neoplasia mucinosa quística (cistoadenoma biliar) es un tumor parecido al estroma ovárico que también se puede presentar como un tumor multiquístico. Ocurre casi exclusivamente en las mujeres y tiene la apariencia de múltiples quistes de paredes bien definidas (quistes dentro de quistes). Estas se pueden asociar a hemorragias y calcificaciones.

El diagnóstico definitivo de Schwannoma se efectúa mediante el estudio histológico e inmunohistoquímico. Microscópicamente se compone de áreas Antoni A y Antoni B. Las áreas de Antoni A son áreas hipercelulares que contienen un gran número de células fusiformes muy compactas. En contraste las células Antoni B es una estructura mixoide con unas pocas células dispersas.

La lesión que describimos en nuestro caso fue quística, el aspecto del tumor depende de la proporción celular Antoni $\mathrm{A} / \mathrm{B}$, las lesiones quísticas tienen una mayor proporción de células Antoni $\mathrm{B}^{20}$.

La inmunohistoquímica resulta útil para distinguirlo de metástasis del estroma gastrointestinal y de otro tipo de tumores ya que muestran marcadores positivos para vimentina y proteína $\mathrm{S} 100$ y son negativos para marcadores musculares, como actina o desmina, así como CD-117 y CD-34, diferenciándose de los tumores estromales gastrointestinales ${ }^{19}$.

En cuanto al pronóstico, dado que la gran mayoría corresponde a lesiones benignas, no afectan la sobrevida del paciente.

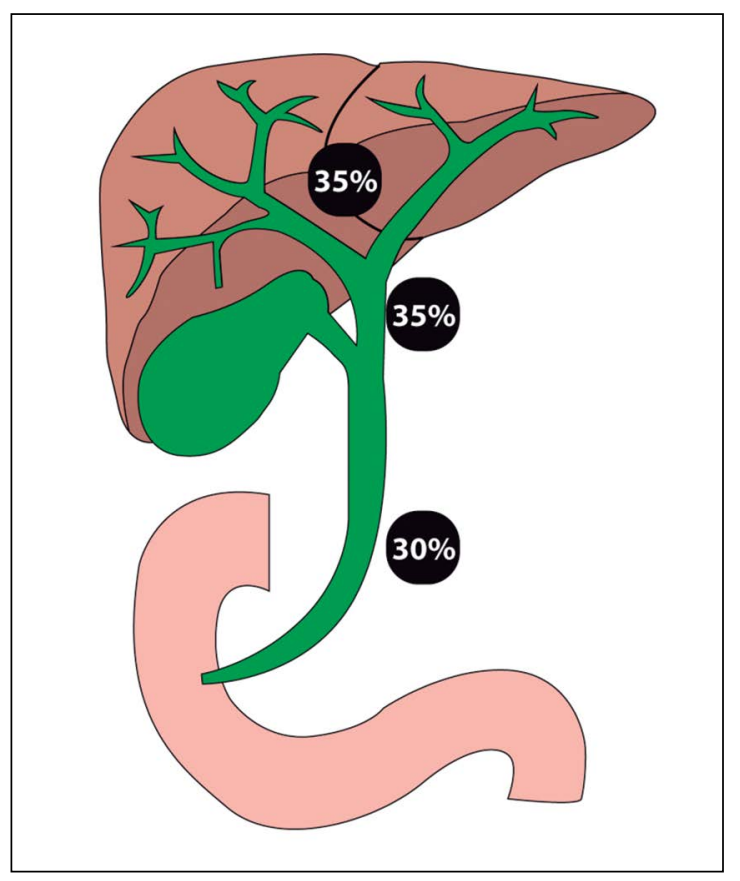

Figura 7. Distribución de la localización de los tumores.

\section{Conclusiones}

Los Schwannomas son lesiones benignas. Suelen confundirse con otras lesiones ya que no tiene características patognomónicas y el diagnóstico es a través del análisis patológico, ya que los Schwannomas son benignos, la resección quirúrgica debería considerarse curativa.

La gran mayoría se diagnostican en el postoperatorio, mediante la biopsia ya que preoperatoriamente son difíciles de diferenciar de otro tipo de lesiones.

\section{Responsabilidades éticas}

Protección de personas y animales. Los autores declaran que para esta investigación no se han realizado experimentos en seres humanos ni en animales.

Confidencialidad de los datos. Los autores declaran que en este artículo no aparecen datos de pacientes.

Conflictos de interés: no hay. 


\section{Bibliografía}

1. Hytiroglou P, Linton P, Klion F, Schwartz $\mathrm{M}$, Miller C, Thung SN. Benign schwannoma of the liver. Arch Pathol Lab Med. 1993;117:216-8.

2. Northover JM, Terblancje J. A new look at the arterial supply of the bile duct in man and it surgical implications Br J Surg. 1979;66:379-84.

3. Whisnant JD Jr, Bennett SE, Huffman SR, Weiss DL, Parker JC, Griffen WO Jr. Common bile duct obstruction by granular cell tumor (schwannoma). Am J Dig Dis. 1974;19:471-6.

4. Balart LA, Hines C, Mitchell W. Granular cell schwannoma of the extrahepatic biliary system. Am J Gastroenterol. 1983;78:297-300.

5. Oden B. Neurinoma of the common bile duct; report of a case. Acta Chir Scand. 1955;108:393-7.

6. Jakobs R, Albert J, Schilling D, Nuesse, Riemann J. Schwannoma of the common bile duct: a rare cause of obstructive jaundice. Endoscopy 2003;35:695-7.

7. Honjo Y, Kobayashi Y, Nakamura T, Takehira Y, Kitagawa M, Ikematsu Y, et al. Extrahepatic biliary schwannoma. Dig Dis Sci. 2003;48:221-6.

8. Otani T, Shioiri T, Mishima H, Ishihara
A, Maeshiro T, Matsuo A, et al. Bile duct schwannoma developed in the remnant choledochal cyst-a case associated with total agenesis of the dorsal pancreas. Dig Liver Dis. 2005;37:705-8.

9. Park MK, Lee KT, Choi YS, Shin DH, Lee JY, Lee JK, et al. A case of benign schwannoma in the porta hepatis. Korean J Gastroenterol. 2006;47:164-7.

10. Vyas FL, Jesudason MR, Samuel R, Govil S, Jesudason SR. Schwannoma of bile duct-a case report. Trop Gastroenterol. 2006;27:50-1.

11. Kamani F, Dorudinia A, Goravanchi F, Rahimi F. Extrahepatic bile duct neurilemmoma mimicking Klatskin tumor. Arch Iran Med. 2007;10:264-7.

12. Fenoglio L, Severini S, Cena P, Migliore E, Bracco C, Fulvio P, et al. Common bile duct schwannoma: a case report and review of literature. World J Gastroenterol. 2007;13:275-8.

13. Jung JH, Joo KR, Chae MJ, Jang JY, Lee SG, Dong SH, et al. Extrahepatic biliary schwannomas: a case report. J Korean Med Sci. 2007;22:549-52.

14. Madhusudhan KS, Srivastava DN, Dash NR, Gupta C, Gupta SD. Case report. Schwannoma of both intrahepatic and extrahepatic bile ducts: a rare case. $\mathrm{Br} \mathrm{J}$ Radiol. 2009;82: e212-5.
15. Kulkarni N, Andrews SJ, Rao V, Rajagopal KV. Case report: Benign porta hepatic schwannoma. Indian J Radiol Imaging 2009; 19:213-5.

16. De Sena G, Molino C, De Riitis MR, Candela S, Cifarelli V, Di Maio V, et al. Surgical management of schwannoma of biliary tract. Chir Ital. 2009;61:119-21.

17. Parameshwarappa S, Rodrigues G, Kumar S, Patil B, Valliathan M. Schwannoma of common bile duct causing obstructive jaundice. Indian J Surg. 2010;72:333-5.

18. Panait L, Learn P, Dimaio C, Do KG, Schwarz T, D'Angelico M, et al. Resection of perihilar biliary schwannoma. Surg Oncol. 2011;20:e1579.

19. Fonseca G, Montagnini A, Rocha M, Patzina R, Vinícius M, Bernardes A, et al. Biliary tract schwannoma: A rare cause of obstructive jaundice in a young patient. World J Gastroenterol. 2012;18:5305-8.

20. Marín C, Valdés A, Alonso I. Schannoma de la vía biliar. Cir Esp. 2015;93:456

21. Haradome H, Woo J, Nakayama $\mathrm{H}$, Watanabe H, Ogawa M, Moriyama $\mathrm{M}$, et al. Characteristics of Hepatic Schwannoma Presenting as an Unusual Multi-cystic Mass on Gadoxetic Acid Disodium-enhanced MR Imaging. Magn Reson Med Sci. 2018;17;95-9. 